\title{
Supporting engagement in research through a game design competition
}

\author{
loanna lacovides* - University of York, UK \\ Anna Cox - University College London, UK \\ Dominic Furniss - Human Reliability Associates, UK \\ Katarzyna Stawarz - University of Bristol, UK \\ Charlene Jennett - University College London, UK \\ Anne Adams - Open University, UK
}

\begin{abstract}
Digital games are an engaging medium that have previously been used for communicating research to a variety of audiences. However, there is an opportunity for engaging people more deeply by involving them in creating games. This article reports on a game design competition, based on participatory design principles and game jam practices, which challenged university students to design games within the context of a research project. Based on their interpretations of research on human error in health care, teams created four games to be disseminated online to a wider public audience. We outline the competition format and reflect on the extent to which it was successful.
\end{abstract}

Keywords: public engagement; participatory design; persuasive games; game design competition

\section{Key messages}

- Participatory forms of public engagement can be achieved through involving members of the public, academics and practitioners in the creation and evaluation of games to communicate research.

- A competition format that emphasizes participatory principles and game jam practices supports participants in creating games that reflect their own interpretations of research.

- A more equitable form of public engagement could potentially be achieved through facilitating further opportunities for two-way acting and listening at different points in the competition.

\section{Introduction}

Alongside traditional research and teaching duties, academics are strongly encouraged to devote time to public engagement activities (Neresini and Bucchi, 2011). Making an effort to engage with the public allows for more open and accessible forms of research (Scanlon, 2014), and can also open up new perspectives and research avenues (Watermeyer, 2012). However, public engagement is often conceptualized 
as a top-down process that focuses purely on 'dissemination, communication or presentation of research' (Grand et al., 2015: 10). Additionally, Sayer and colleagues (2014) note that it is seen as a challenging activity, and argue that there is a need for more creative and equitable approaches.

Previously, digital games have been used to communicate research to members of the public (for example, Ouariachi et al., 2017), but there is potential to increase participation through involving people more directly in creating these games. As with participatory forms of research, the democratization of design allows for the participation of users in decisions that will affect them, as well as opportunities for learning and collaboration (Kelty et al., 2015). Certain principles are often emphasized as underlying the participatory design process and have provided motivation for its use. These include sharing of control with users to empower them (Frauenberger et al., 2015; Vines et al., 2013), ensuring that users can gain or learn from participation (Bossen et al., 2010) and supporting mutual learning between users and designers (Halskov and Hansen, 2015).

\section{Participatory game design}

Within formal educational contexts, participatory game design has been viewed as a way to produce engaging games, where the students involved are able to decide 'what is fun or not' (Danielsson and Wiberg, 2006: 275). For example, Danielsson and Wiberg (2006) describe the development of a game about gender issues (called 'His and Hers') that involved Swedish teenagers. However, following Druin (2002), they describe their participants as 'informants' (rather than 'design partners') because, while they contributed to game content and design decisions early on in the process, they were not directly involved in creating sketches or prototypes. Similarly, Lukosch and colleagues (2012) describe the development of a simulation game for supporting social awareness training for police officers, which included interviews to elicit requirements and playtesting of prototypes with six professionals. Following Gulliksen et al. (1999), the authors define participatory design as 'a design approach in which the users actually participate in and are in charge of the making of the design decisions' (Lukosch et al., 2012: 4). However, the process they describe seems much closer to user-centred design, where participants act as testers and control appears to remain with designers.

The reduced level of involvement in the design processes described above arguably leads to fewer opportunities to engage with a particular domain and to develop additional skills. The previous examples also suggest, within the context of participatory game design, that there is a strong focus on the outcomes produced and the extent to which they can serve as effective and engaging learning tools. However, despite research on constructionist gaming indicating that another way to engage learners is to task them with creating games themselves (Kafai and Burke, 2016), there has been less consideration of how participants can learn from engaging directly in the design process. Therefore, we look to other game design formats that provide opportunities for engagement and learning, and that can also empower participants within the development process.

\section{Game jams}

Game jams are hackathon-style events for game development that have become increasingly popular (Fowler et al., 2013). Musil et al. (2010) describe them as events that occur over short periods of time, where small teams work together to rapidly 
prototype game ideas around a particular theme to win prizes awarded by judges or peers. They can differ in scale from small, one-off meet-ups that occur in a single location, to international, annual events, where teams submit their prototypes online (such as the Global Game Jam or Ludum Dare). While they are often set up as competitive events, the main motivations for taking part tend to be intrinsic, where participants want to practise and learn about creating games through collaborating with others (Reng et al., 2013).

Musil et al. (2010) refer to participatory design as a component of game jams, as the events bring together multidisciplinary teams (including designers, artists and developers), and there is a lack of prescribed roles, which enables a safe space for idea generation. However, while both participatory design and game jams emphasize sharing control and learning through collaboration with people with different skills, knowledge and experience, there are some key differences between the two. First, game jams are normally quite short events, and while there have been some attempts to embed them in wider research or design projects (for example, Shapiro et al., 2014), this is not usually the case. Second, game jams emphasize the autonomy of teams, providing design constraints for focus, but allowing participants the freedom to make their own decisions (Goddard et al., 2014). While control in participatory design is meant to be shared among the participants and designers, this does not always occur and, due to challenges such as lack of technical skills or domain knowledge (Khaled and Vasalou, 2014), participants are rarely responsible for development tasks. Third, while there has been increasing interest in using game jams for particular purposes, for example for research (Deen et al., 2014) or to create exercise games (Moser et al., 2014), they tend to result in prototypes, rather than fully operational games.

Despite the benefits that game jams can provide to participants, their timelimited nature means it is less common for game development to continue beyond the end of the event (Goddard et al., 2014; Ferrario et al., 2014). Longer-term competitions or summer schools (Jennett et al., 2016) may be a way around this issue, though there are few examples of participatory design competitions. While Lam (2013) does report on a project where secondary school students were challenged to design games around the theme of promoting community improvements, in this case the students were only given two days to come up with design ideas, which were then passed on to professional designers. As with other approaches to participatory game design, the format adopted in this case also appeared to support a more limited form of involvement where participants did not have a large amount of control over the development process.

\section{Our approach}

In developing our competition format, we looked to participatory design approaches and game jam practices to create a more equitable form of public engagement. Our aim was to engage members of the public in the process of designing games that could be hosted online as part of the dissemination of research to a wider audience. By providing opportunities to engage in a dialogue around our research, and by allowing participants to create their own interpretations, the format emphasized participatory principles of sharing control (Frauenberger et al., 2015) and providing opportunities for learning (Bossen et al., 2010; Halskov and Hansen, 2015).

As our goal was to create games for young people not familiar with the domain, and to involve them in the development process, we recruited university students to take part in the competition. In addition to being a similar demographic to our 
target audience (young people who at least occasionally played games), the approach enabled us to target those interested in developing game design and development skills that they could go on to use in future. Furthermore, previous research with university students illustrates how they retain multiple identities, making them not only members of a university but also members of various different publics (Adams, 2013).

In this article, we present a descriptive case study (Yin, 2009) of an approach to designing games for public engagement. The evaluation of the competition entries is described in detail in lacovides and Cox (2015). The current article builds upon previous work by reflecting on the strengths and weaknesses of the competition format and discussing the extent to which the competition met our goals. Additionally, we reflect on the participatory nature of the approach and how the format could be amended in future.

\section{The game design competition}

\section{Background and motivation}

The competition was organized as part of the CHI+MED research project, which investigated ways to improve safety within health care. The competition challenged teams to develop a game that would raise awareness and lead to reflection on human error and blame culture within the context of health care, that is, a game that got players thinking about how individuals get blamed when, in fact, it is the wider system that is at fault. For example, when a disaster strikes, the media often look for an individual to blame, whether this is the pilot of an airliner that crashed or the nurse who delivered a fatal dose of medication. This focus on the individual and their punishment or removal is indicative of a 'blame culture' (Dekker, 2012). Researchers argue that we should make efforts to move from a blame culture in health care to a culture that is 'just', which tries to foster learning rather than punitive actions on individuals (Khatri et al., 2009). Doing so involves focusing on issues at a system level, rather than the active errors of the individuals involved (Reason, 2000).

Within $\mathrm{CHI}+\mathrm{MED}$, different mediums for public engagement were explored, for example, social media, science fair exhibits and games. For instance, the Twitter hashtag \#errordiary was started as a way to encourage individuals to share day-today errors and raise awareness about their prevalence. The range of errors collated have been used to challenge notions that medical error is somehow different to the everyday errors we all make (Furniss et al., 2014). However, an initial interview study suggested that the majority of Errordiary volunteers are researchers who are already interested in human error research (Jennett et al., 2014). This finding highlighted the need to explore new ways of engaging wider audiences in the human-error debate.

Therefore, we looked to digital games as a way to engage people, not just through playing them, but also through the process of making them. We invited a broad range of university students to compete in a game design competition where the winning entries would be hosted on the project's public engagement website (Errordiary.org). We challenged teams to develop a game, based on our research, which would inspire curiosity and reflection on human error and blame culture.

\section{Competition overview}

The competition ran over a four-month period to provide enough time to complete the design process and lead to fully functioning games. The process began with an initial kick-off day and ended with a prize-giving and a showcase. Teams could consist 
of between two and six members, with at least one team member who attended the initial kick-off. All team members had to be students (at any level of higher education, from any institution, and in any subject area), and cross-disciplinary collaboration was strongly encouraged. The game needed to be a web application (for example, HTML/ JavaScript) that would be playable in all major web browsers. The teams were allowed to use game engines, such as Unity. After the kick-off, the teams had two months to complete and submit their games.

\section{Ethical considerations}

Our research activities were approved by our departmental ethics panel as per our standard practice. In this section we consider additional issues relating to the use of cash prizes and copyright. While we were aware that the inclusion of large cash prizes can be somewhat controversial in hackathon-style events (Elias, 2014), and that the motivations of those who participate in game jams tend to be intrinsic (Reng et al., 2013), we did not want potential participants to think that we were trying to crowdsource their efforts for free. Thus, we offered the following prizes: $£ 1,000$ for first place, $£ 500$ for the runner-up, and $£ 500$ for a People's Choice award voted at the final showcase. Given that the prizes were likely to be split across at least two team members, we felt that the amount was not so large that it would attract people who were only interested in earning money.

From the start of the competition, we made clear that the games produced would be made freely available on Errordiary.org. Furthermore, they were to be licensed under a Creative Commons Attribution-Noncommercial 4.0 International License to ensure that the creators retained copyright, while others would be free to adapt them (as long as the original creators were given credit and any derivatives were not produced for commercial purposes). This information was communicated via the competition website and during the kick-off day, while the submission form made clear that by submitting the game, the participants were agreeing to these conditions.

After the competition, we gave the teams an opportunity to provide feedback on the descriptions that would accompany the games on the website, which included listing each of the individuals involved by name. We wanted to ensure that team members were given credit for creating their game, and also gave them the option to add links to their own websites or online profiles.

\section{Kick-off event}

The competition started with a kick-off day, which introduced attendees to the competition goals and themes, provided them with opportunities to learn from and engage in dialogue with the different domain experts, allowed them to brainstorm initial design ideas, and attempted to support the formation of teams. The event was publicized as a 'Persuasive Game Design Competition' via posters, mailing lists and social media across different universities, departments, courses and student societies in and around London, UK (where the kick-off was to be held). In addition to the CHII+MED project partners in London (three universities), over thirty institutions were contacted, with a focus on targeting computer science, game design, and art and design departments, as well as student groups interested in games. We highlighted 'persuasive games' in the competition name to emphasize the need for the games to introduce new concepts and challenge the ways in which players view the world (Bogost, 2007). 
Similar to the start of a game jam, the day started with an introduction to the competition, followed by a series of mini-talks from four experts in human error, blame culture, health care and game design. The first two experts were involved in the $\mathrm{CHI}+\mathrm{MED}$ project, and spoke about their research areas; the third, a practising nurse, focused on the experience of working in health care, and how errors occur and are dealt with; the fourth, a game designer, spoke about designing persuasive games. Talks were followed by a Q\&A panel with all the speakers.

After lunch, we held a game design workshop run by the game designer, with assistance from the domain experts, who acted as mentors. The workshop included an overview of how to design games, including examples from the game designer's own practice, emphasizing the importance of rapid prototyping and testing with users. The students then worked in randomly assigned groups, with materials such as dice, board game pieces, Lego, and pen and paper, to help them start brainstorming possible game ideas (see Figure 1). The day concluded with a networking session that was intended to facilitate team formation.

\section{Figure 1: Participants within the game design workshop prototyping initial game ideas}

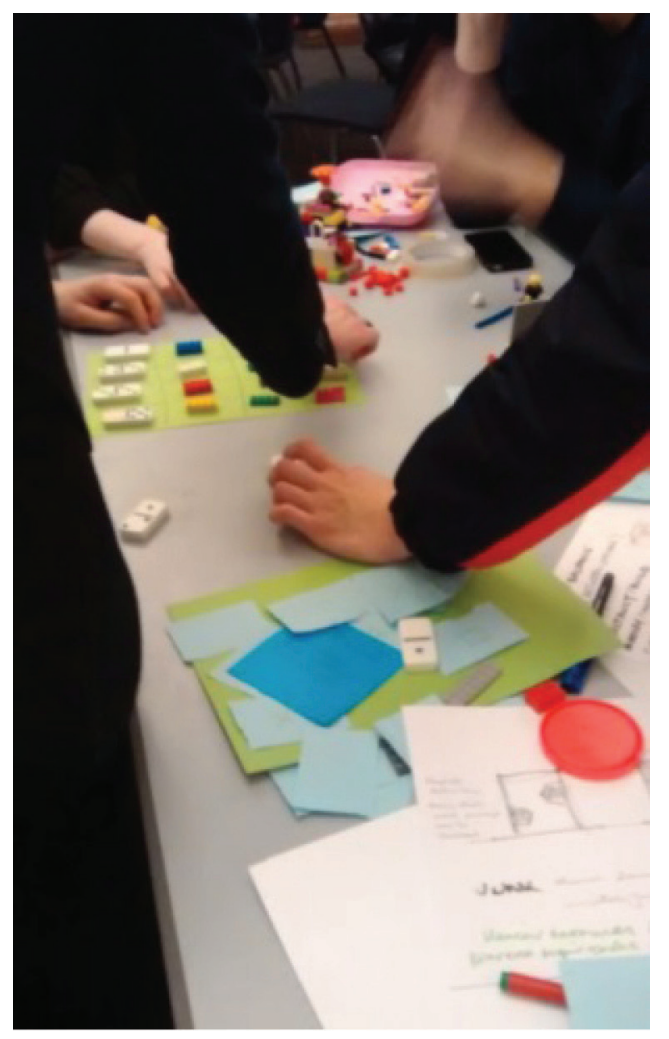

Credit: loanna lacovides

Given the importance of communication within participatory approaches (Iversen et al., 2010), the Q\&A panel, game design workshop and networking sessions (along with lunch and coffee breaks) were all included to ensure the students had opportunities to interact with the domain experts and each other. We wanted to ensure that students would be given relevant information about research and practice for their games, while domain experts could gain insights from the students relating to game ideas and their understanding of human error. Additionally, we set up forums so the students 
would be able to discuss issues and communicate with the experts, and a Facebook group to support team formation after the event. The competition was also supported through a website, which contained important information (such as the brief, rules and deadlines) and further sources of inspiration (such as examples of persuasive games, game design resources, information relating to human error and personal accounts of working in health care).

\section{Competition entries}

The submission form asked for a brief description of the game and for an explanation of how the game was designed to make people think about human error and blame culture. The form also included questions about the roles of each team member within the development process, and how they tested the game with other players. These forms allowed us to gather insight into what they had learnt from taking part in the competition, thus informing and deepening understanding of this approach to public engagement.

Nine student teams registered for the competition, and four submitted entries. In total, 12 people took part in the competition, 8 of whom attended the initial kick-off. Each team consisted of between 2 and 4 students. A mixture of undergraduates and postgraduates took part, from computer science, communication, psychology and medicine departments across five UK universities (only one of which was part of the $\mathrm{CHI}+\mathrm{MED}$ project). Their entries (hosted on http://bit.ly/ErrorGames) are described below in alphabetical order:

- Medical Student Errors was created by Devon Buchanan and Angela Sheard. The game is an interactive fiction about a day in the life of a junior doctor. Through a text-based interface, the player is presented with a number of scenarios related to how people make and communicate errors. Using hyperlinks, the player can move backwards and forwards through the narrative, exploring dialogue options and finding out more about particular concepts.

- Nurse's Dilemma was created by Adam Afghan, Andrew Gorman, Natasha Trotman and Jining (Kea) Zhang. The player is cast in the role of a nurse faced with a series of challenges during her daily tasks. The game uses a text-based interface with simple audio and graphics. The designers describe it as an empathy-based game that aims to shed light on the pressures, constraints and stresses that nurses are expected to deal with on a day-to-day basis.

- Patient Panic was created by Cameron Kyle-Davidson, Lydia Pauly, Benjamin Williams and Connor Wood. The game is set during a natural disaster, and the player is a local doctor who has to treat a multitude of patients before it is too late. As in Tetris, there is no win state: the game gradually increases in difficulty until the player runs out of lives and is fired due to their inability to cope. In addition to offering three levels of difficulty, the game employs a simple pointand-click interface, animations and a soundtrack involving ambulance sirens.

- St. Error Hospital was created by Charmian Dawson and Subhan Shaffi. The game adopts a bird's-eye view of a hospital where players take on a management role - balancing a budget, directing staff, organizing ward areas and implementing 'resilience strategies' that aim to reduce the likelihood of errors. The player can choose between two levels of difficulty. The game also displays information reports and graphs to provide feedback on the player's performance. In terms of audio, a background hum is present throughout the game to indicate ward activity. 


\section{Evaluation of the entries}

A summative evaluation was carried out to establish the winners and assess whether the games were suitable to share online, involving a combination of expert judging and playtesting. The expert judging consisted of six judges with expertise in human error, health care, game design and usability, including the competition chair, two of the experts from the kick-off day, and three researchers from the university where the kick-off was hosted. Three of the judges, including the chair, were also part of the competition-organizing committee and were involved in the $\mathrm{CHI}+\mathrm{MED}$ project.

The playtesting involved 12 participants ( 9 female; 3 male) recruited from a university participant pool. They played each game (where the order was counterbalanced) for up to ten minutes, and answered a short questionnaire on each before a final post-play interview. The evaluation was primarily concerned with evaluating the 'serious experience' (Marsh and Costello, 2013). Marsh and Costello (ibid.) highlight the importance of establishing the extent to which serious experiences 'linger' with players, so participants were also sent questions via email two days after the sessions to investigate whether any of the games resonated with players. While we provide an overview of the findings below, the combination of methods developed and the outcomes of the evaluation are described in detail elsewhere (lacovides and Cox, 2015).

The evaluation methods we developed allowed for a consideration of domain relevance and the potential to promote reflection (expert judging), gameplay experience and engagement with competition themes (playtesting and interviews) and longer-term resonance (follow-up emails). On this basis, Nurse's Dilemma was awarded first prize, with St. Error Hospital as runner-up. Nurse's Dilemma created a particularly compelling experience where the game enabled empathy with nurses and an understanding of how tensions within a system can affect individuals. Although St. Error Hospital was a close second, the complexity of the game unfortunately meant that players were not always able to connect the gameplay to a consideration of the competition topic. The winners were announced at a final prize-giving and showcase, where the teams were invited to give attendees an opportunity to try out their games, and to present an overview of their game and the design process. Nurse's Dilemma was also voted the People's Choice by the audience.

To maximize the learning potential of taking part in the competition, teams were provided with summary feedback based on the judging and playtesting sessions, which explained the evaluation process and highlighted the strengths and weaknesses of their game.

\section{How successful was the competition?}

Our goal was to engage members of the public and domain experts in the process of designing games that could be hosted online to communicate our research to a wider audience. To address the question of the extent to which this was successful as an engagement approach, we will first briefly discuss the games as outcomes produced by the competition, before considering the process of how they were developed.

\section{The competition outcomes}

The evaluation process revealed that the judges and participants had their own preferences concerning which games they liked and what they got from them. Given the differing views, and the quality of all four games submitted, we made all of them 
available online to showcase the different ways in which the teams approached the competition challenge and interpreted our research. Since then, the game pages have had a combined total of 2,038 unique page views, with the average visit time being 2 minutes 33 seconds. While the evaluation suggested that, to differing degrees, the games were successful at getting young adults to reflect on human error and blame culture (lacovides and Cox, 2015), the analytics data do not contain any demographic information concerning the age of those who played the games online. Thus, it is difficult to establish the extent to which the games were able to reach our intended audience. However, we argue that the fact that the competition led to four different, yet relevant and well-thought-out, games suggests this approach was successful at producing playable and potentially impactful outcomes.

After the competition, the CHI+MED project was also contacted by a nurse trainer about using Nurse's Dilemma. The trainer used the game as a preparatory activity for a training session in Hong Kong that focused on leadership. While healthcare practitioners were not considered the initial audience for the games produced, it seems there is potential to use them within more formal educational contexts to promote discussion and support learning.

\section{The competition process}

Overall, 31 students were involved in at least some parts of the competition: 27 students attended the kick-off, 24 of whom later registered (across nine teams) to take part. By the end of the competition, participation decreased, with only four teams submitting a game. Eight experts were also involved, participating in the kick-off day and/or the judging process. Finally, approximately 35 people (a mix of people from the wider research project and the wider public) also attended the final showcase.

Although we were aware that participation was likely to drop off, we considered the whole competition as an exercise in engaging different people at different points: attending the kick-off event, developing a game, attending the showcase and, finally, playing the games online. As such, although the competition did experience relatively high attrition rates, we were able to introduce a variety of people to the concepts of human error and blame culture - potentially more so than if we had focused primarily on involving a small number of people in workshops (as can be the case in participatory design for serious games; see, for example, Danielsson and Wiberg, 2006; Vasalou et al., 2012).

With respect to kick-off attendees, 13 (4 female, 9 male; mean age: 24.4 years) out of 27 filled in evaluation forms, which suggested that people enjoyed the event overall and thought that they had learnt from the experience. For instance, one student remarked:

The information about human error and the effects of blame culture was really interesting, I learnt a lot and am interested to find out more. The strategy for game prototyping was also useful and something I think I could use in future.

Another student stated, 'the [game design] workshop was a great way to connect with new people and start brainstorming about ideas'. Others commented on the quality of the talks - for example, 'The human error talk was very good, especially the example cases given to demonstrate the consequences of human error' - and provided ideas about how to improve the event - for example, 'Shorter talks, longer Q\&A, longer workshop'. During conversations between the experts, it also became clear that they 
had learnt from taking part, particularly in relation to the topics they were less aware of (game design, for example), and from discussing game ideas with the students.

The least successful part of the kick-off related to the networking session, as many students had already decided who they would be working with. Only two people used the Facebook group to join a team, but although one dropped out, the other remained in the competition and submitted a game. Additionally, the forums, set up to facilitate communication with experts after the kick-off, were not used at all. While it may be that the teams did not feel they needed to engage in further discussion, or perhaps did not wish to do so on a public forum within the context of a competition, the general lack of activity across the Errordiary forums as a whole could also have been discouraging.

Despite the lack of continued communication, the submission forms and the presentations at the final prize-giving can be viewed as evidence that the teams had been inspired by expert accounts, and had engaged with many of the resources on the website. For example, the Nurse's Dilemma submission form referred to a blog post describing the experience of being a nurse and the game Hush, which was listed as an example of a persuasive game on the competition website. Furthermore, there was evidence of learning, as teams appeared to take on board advice about prototyping and organizing their own formative playtesting. For example, Medical Student Errors was tested as a prototype with two individuals outside the development team, while Patient Panic was shared on Facebook and got feedback from 10-15 players. The games themselves also provide an indication of what the students learnt about the competition themes and game development.

\section{Tensions that emerged}

While the evaluation indicated that the games were able to meet the challenge criteria, certain issues did emerge over the course of the competition that led to reflection and debate among the experts involved. The first related to some of the interpretations that were made. For example, in academic terms, resilience strategies are informal strategies developed by individuals, often outside official practice, rather than procedures implemented by management (Furniss et al., 2011). However, the way the concept is presented in St. Error Hospital suggests that the designers understood them as the latter.

The second challenge involved having to reconcile different opinions. For instance, one judge was concerned that Nurse's Dilemma was somewhat over the top in terms of how it depicted working in a hospital, and a couple of the playtesters questioned how likely the experience was. However, another of the judges who was a nurse, along with a colleague who had also played the game, agreed that it was a realistic interpretation of their own experiences. During the playtesting, there was also sometimes a tension around player expectations of gameplay and the experience of playing persuasive games about serious issues.

One response to these tensions is to suggest that a greater amount of expert involvement in the design process is required to ensure that participants will create an accurate representation of the problem area. However, this would reflect a top-down approach where we assume that knowledge is something that can only be 'transferred' from experts to the public (Grand et al., 2015), a perspective we were keen to avoid. In addition, the competition challenged students not to deliver particular learning content but to inspire reflection and curiosity around the competition themes in a compelling way. Thus, we were less concerned with whether players wanted to play a game multiple times or how 'fun' gameplay was, and more concerned with which 
game experiences (even as a one-off) were likely to get players thinking more broadly about human error and blame culture within health care.

An alternative interpretation is that designers should be allowed the freedom to make their own interpretations, and to take some artistic licence, in order to communicate a particular message or experience. In the case of Nurse's Dilemma, regardless of debate between the judges about the accuracy of the portrayal, the evaluation indicated the game was most likely to achieve the competition goal. Furthermore, it is perhaps through the subverting of expectations that reflection was made more likely. Although tensions did occur, we suggest that the format allowed the teams to interpret our research in creative ways. Additionally, the tensions themselves actually served as useful points of reflection for the research team.

\section{Discussion}

Even though the National Co-ordinating Centre for Public Engagement defines public engagement as 'a two-way process, involving acting and listening, with the goal of generating mutual benefit' (NCCPE, 2018), public engagement is often conceptualized by academics as a one-way, top-down process that focuses on 'dissemination, communication or presentation of research' (Grand et al., 2015: 10). While we were keen to avoid the latter through adopting a more participatory approach to designing games, we recognize that the competition itself did not go far enough in terms of encouraging a two-way engagement process.

To engage reflexively with our approach, we address three questions proposed by Vines and colleagues (2013) in relation to adopting participatory approaches. In doing so, we also consider the extent to which the competition was able to meet the NCCPE criteria.

\section{Who initiates, directs and benefits from user participation?}

This question relates to the concept of 'mutual benefit' (NCCPE, 2018). Participation was initiated and directed by members of the research project, who were interested in exploring more creative and equitable ways to support the use of games within a public engagement context. In this case, our users were players, and our intended audience for the games was young adults who were not familiar with human-error research. We also needed participants with at least some experience in design and development practices, so we recruited university students from a range of related departments and student groups. The competition was entirely voluntary and participants could drop out at any time.

We argue that everyone involved benefited from the competition in one way or another. As a research team, we benefited from the students' game development skills and gained a set of games to host online, thus expanding our public engagement and research portfolios. The games also allowed us to question how we define topics from our own research (resilience strategies, for example). Our student participants were provided with multiple opportunities to learn about a new domain (through the kick-off, the resources provided, operationalizing particular concepts in their games and being supplied with evaluation feedback) and to develop skills in game development (including prototyping, user-testing and working in a team). In addition, the winning teams earned some prize money. Those who attended the competition events, particularly the kick-off, also seemed to benefit in terms of learning about unfamiliar domains and through being provided with opportunities to network with other people (regardless of whether they entered the competition or not). The reuse 
of the game by domain experts for training purposes also highlights a cyclic effect of engagement leading into impact, with the engagement of students, domain experts and the research team creating further opportunities for wider engagement with the general public and domain experts outside the research team.

\section{In what forms does participation occur?}

The competition could be described as a form of 'creative citizenship' where the aim was 'not to influence public debate of formal political processes through rational discourse but to create a collective experience involving creative expression, listening and collaboration' (Mahony and Stephansen, 2016: 590). However, while participation occurred in a range of forms across the competition, the 'acting and listening' (NCCPE, 2018) that occurred was rarely two-way.

The kick-off event did attempt to facilitate the brokering of knowledge between the research team and potential participants, where the domain experts and students participated through giving and listening to talks, asking and answering questions, and taking part in the game design workshop. However, while we tried to facilitate opportunities for communication through including a Q\&A session, tea/coffee/ food breaks and hosting a game design workshop, the general emphasis was on the participants listening to the experts to find out more about the domain and the competition. While we had hoped that further communication might occur between the two groups after the kick-off via the forums, unfortunately this was not the case.

Participation after the kick-off included the student teams making and submitting games, and then the experts conducting a summative evaluation of the games by judging them and running playtesting sessions. We did not involve the student designers in the summative playtesting, as the competition format would have given rise to a conflict of interest. At the prize-giving, students were able to see competing games and showcase their entries by presenting an overview of their design process to an audience who participated by voting for their favourite game. The research team also put together a summary of the evaluation findings to communicate feedback to each team so they could learn from the evaluation findings. Finally, the games are now online and freely available for others to play.

Although the competition did involve multiple points of participation, twoway acting and listening would have required both additional opportunities for communication between the domain experts and the participants during development, and clear evidence that the interaction with the participants had directly influenced the research and working practices of those involved in organizing the competition.

\section{How is control shared with users in design?}

While the competition may not have resulted in a clear 'two-way' form of engagement (NCCPE, 2018), the format did allow us to avoid a purely top-down process through providing the student teams with control over development. In effect, control was handed back and forth between the participating teams and the researchers. The competition themes were prescribed by the organizers, who had initial control over structuring the competition. After the kick-off, teams then had complete power over how to develop their games, and were also free to drop out of the competition. This handing over of control to the student teams is less common in many instances of participatory game design, where participant engagement tends to be limited to the informant level. Although the kick-off and website resources served to inspire the 
students, it was up to each team to form their own interpretations of our research, and to decide collectively how to approach the competition challenge.

While handing over creative control was intended to empower the teams and lead to greater learning (Li, 2010; Kafai and Burke, 2016), it also meant that we did not know what sort of games we were going to get. There was also a risk that no one would take part or produce games that addressed the competition challenge. However, as organizers of the competition, we also had the final say, and could have vetoed anything we felt was wildly inaccurate or even offensive. The students would still have been free to do what they liked with their games, but they would not have won the competition, and the games would not have been hosted online on the public engagement website. So, while we gave participants control over development, we still had some power in terms of what happened after submission.

\section{Future directions}

The competition format we developed could be adapted by others wanting to adopt a participatory approach to engagement when creating games to communicate research. The process could also be amended to support further opportunities for two-way forms of engagement.

While our research focused on human error in health care, there is scope to apply the approach across a range of domains. When doing so, as suggested by Goddard and colleagues (2014) in relation to game jams, the theme or challenge should aim for a balance between specificity (to serve as a design constraint) and ambiguity (to allow for autonomy and creativity). There is potential here to make the process more two-way from the start by reaching out to particular groups or audiences and including them in discussions about the theme and goals of the activity. Furthermore, it is important to ensure that any claims about copyright are made transparent in advance.

In terms of recruitment, drop-off is an important issue to consider. Extensive effort needs to be applied at the early stages to publicize the competition and ensure a large set of people attend the initial kick-off. That said, the higher the number of participants, the more resources required to support the process. In addition to including a range of domain and design experts, a diverse set of participants should be recruited, so that teams contain a mix of different skill sets and can engage in multidisciplinary collaboration. Consideration should also be given to face-to-face and online networking opportunities to support those without a team or to help teams who need a specific skill set. Furthermore, including both a kick-off and showcase event, as well as sharing the games online, allows for multiple opportunities to engage others who may not have the time to take part in development.

Another way to decrease attrition and facilitate more two-way forms of communication would be to ask the participants what channels they would prefer to use and how often they would like to do so. Decisions would need to be made about whether these channels should be public (for example, weekly forum discussions), private (for example, scheduling a Skype session), or a mixture of both. While in our case time constraints did not allow for another round of testing, the comments from judges and playtesters could also be fed back to the designers as part of a formative evaluation.

Additionally, the proliferation of design tools such as GameMaker, Scratch and Twine suggests that there is scope to involve different audiences, such as younger participants and/or those without design or development skills. Although participants may still need a level of support, these types of tool do not require programming 
expertise and have made game making more accessible to a wider audience (Resnick et al., 2009). Alternatively, participants can focus on developing board games, where there is potential to share the outputs through creating them in a format that allows for 'print and play'. Another key consideration is how to promote the games to ensure they reach their intended audience, something we could have done in a more targeted way.

In terms of evaluation, it is also worth considering both output and process. While our evaluation suggests that the games had an impact on individuals (lacovides and Cox, 2015), the fact that the games were disseminated online means it is harder to establish the extent to which they reached our target demographic. The use of online surveys or more refined analytics could provide more detailed evidence in this respect. Furthermore, post-competition interviews with student team members could allow for further exploration of how the process impacted individuals. Similarly, while we have adopted a reflexive approach within this paper, there could have been a more explicit investigation of the experience of the research team and experts that took part, for example in terms of how participation might have influenced their subsequent research and practice to provide evidence of two-way engagement. A longer-term evaluation with all those involved could have helped to indicate whether any 'ripple effects' led to benefits over time (Reng et al., 2013).

\section{Conclusion}

We present an approach to public engagement based on participatory approaches and game-jam practices. The competition included a kick-off event, judging and final showcase, while emphasizing the participatory principles of sharing control and providing opportunities for learning. We argue that the format adopted allowed us to avoid a purely top-down approach, where we could involve members of the public, academics and practitioners in creating games to communicate research. However, despite providing multiple points of participation, we recognize that the competition could have gone further in achieving a more equitable type of engagement. Through taking a reflexive approach to our work, we suggest ways in which the structure could be adapted to facilitate two-way forms of acting and listening, thus increasing the potential benefit for all those involved.

\section{Acknowledgements}

We would like to thank all the teams that took part in the competition, the participants from the playtesting sessions, the domain experts and judges. Special thanks to members of $\mathrm{CHI}+\mathrm{MED}$ who took part in discussions about the competition, including Sandy Gould who incorporated the games into Errordiary.org. The authors were supported by the EPSRC-funded CHI+MED project (EP/G059063/1), and EU project Citizen Cyberlab (317705).

\section{Notes on the contributors}

Dr loanna (Jo) lacovides is a lecturer at the University of York. Her research interests lie in human-computer interaction and educational technology, with a focus on investigating learning within the context of games and playful technologies. She has explored how people learn to use technology in the workplace, digital technologies and informal learning, and the use of digital games in formal education. 
Professor Anna Cox is Deputy Director at the UCL Interaction Centre and Professor of Human-Computer Interaction. Her research is motivated by an interest in the impacts of digital technologies on how people get work done, how they manage their work-life boundaries, how they spend their leisure time, and supporting health and well-being. Anna co-led CHI+MED, an EPSRC-funded project to improve the safety of interactive medical devices.

Dr Dominic Furniss is a Human Factors Consultant at Human Reliability Associates. $\mathrm{He}$ is interested in understanding sociotechnical systems: how people, policies, processes and products interact to impact quality and safety. Interests include human error, usability, resilient systems, and public and patient involvement.

Dr Katarzyna Stawarz is a research associate at Bristol Interaction Group, working on the NIHR-funded INTERACT project, which aims to develop and evaluate an intervention for delivering high-intensity cognitive behavioural therapy for depression. She completed her PhD at the UCL Interaction Centre, and her research investigated the use of smartphone apps in developing habits and reducing medication nonadherence. She has a keen interest in digital health, and in inclusive and ethical design.

Dr Charlene Jennett was a research associate at the UCL Interaction Centre. She received her PhD in human-computer interaction in 2010. Her research interests include human-computer interaction, digital games and citizen science. She most recently worked on the EPSRC-funded Open 3D project, investigating users' experiences of citizen science.

Dr Anne Adams is the Open University Associate Director for Professional Development and Director for Knowledge Exchange in the Centre for Policing Research and Learning. She has coordinated large-scale international research projects in technology-enhanced learning, the $€ 2.1$ million EU Juxtalearn project, and the knowledge exchange RCUK Catalyst project. She has over a hundred publications (H-Index 20), with an expertise in educational technology, human-computer interaction, work-based collaboration and secure systems development.

\section{References}

Adams, A. (2013) 'Situated e-learning: Empowerment and barriers to identity changes'. In Warburton, S. and Hatzipanagos, S. (eds) Digital Identity and Social Media. Hershey, PA: Information Science Reference, 159-75.

Bogost, I. (2007) Persuasive Games: The expressive power of videogames. Cambridge, MA: MIT Press.

Bossen, C., Dindler, C. and Iversen, O.S. (2010) 'User gains and PD aims: Assessment from a participatory design project'. In Proceedings of the 11th Biennial Participatory Design Conference. New York: Association for Computing Machinery, 141-50.

Danielsson, K. and Wiberg, C. (2006) 'Participatory design of learning media: Designing educational computer games with and for teenagers'. Interactive Technology and Smart Education, 3 (4), 275-91.

Deen, M., Cercos, R., Chatman, A., Naseem, A., Bernhaupt, R., Fowler, A., Schouten, B. and Mueller, F. (2014) 'Game jam: [4 research]'. In CHI '14 Extended Abstracts on Human Factors in Computing Systems. New York: Association for Computing Machinery, $25-8$.

Dekker, S. (2012) Just Culture: Balancing safety and accountability. 2nd ed. Farnham: Ashgate Publishing.

Druin, A. (2002) 'The role of children in the design of new technology'. Behaviour and Information Technology, 21 (1), 1-25.

Elias, J. (2014) 'Six things experienced hackathon planners know (that you don't)'. Fast Company, 3 April. Online. https://tinyurl.com/ybkhrqd9 (accessed 4 November 2018). 
Ferrario, M.A., Simm, W., Newman, P., Forshaw, S. and Whittle, J. (2014) 'Software engineering for "social good": Integrating action research, participatory design, and agile development'. In Companion Proceedings of the 36th International Conference on Software Engineering. New York: Association for Computing Machinery, 520-3.

Fowler, A., Khosmood, F. and Arya, A. (2013) 'The evolution and significance of the Global Game Jam'. In Workshop Proceedings of the 8th International Conference on the Foundations of Digital Games, Chania, Crete, Greece, 14-17 May 2013. Online. www.fdg2013.org/program/ workshops/papers/GGJ2013/ggj13_submission_1.pdf (accessed 2 December 2018).

Frauenberger, C., Good, J., Fitzpatrick, G. and Iversen, O.S. (2015) 'In pursuit of rigour and accountability in participatory design'. International Journal of Human-Computer Studies, 74, 93-106.

Furniss, D., Back, J., Blandford, A., Hildebrandt, M. and Broberg, H. (2011) 'A resilience markers framework for small teams'. Reliability Engineering and System Safety, 96 (1), 2-10.

Furniss, D., lacovides, J., Jennett, C., Gould, S., Cox, A. and Blandford, A. (2014) 'How to run an Errordiary workshop: Exploring errors and resilience strategies with patients, professionals and the public'. Paper presented at the 3rd Resilient Health Care Net (RHCN) Meeting, Hindsgavl Castle, Denmark, 12-14 August 2014.

Goddard, W., Byrne, R. and Mueller, F. (2014) 'Playful game jams: Guidelines for designed outcomes'. In Proceedings of the 2014 Conference on Interactive Entertainment. New York: Association for Computing Machinery, 1-10.

Grand, A., Davies, G., Holliman, R. and Adams, A. (2015) 'Mapping public engagement with research in a UK University'. PLoS One, 10 (4), Article e0121874, 1-19. Online. https://tinyurl.com/ y929dcqk (accessed 4 November 2018).

Gulliksen, J., Lantz, A. and Boivie, I. (1999) 'User centered design - problems and possibilities: A summary of the 1998 PDC and CSCW workshop'. SIGCHI Bulletin, 31 (2), 25-35.

Halskov, K. and Hansen N.B. (2015) 'The diversity of participatory design research practice at PDC 2002-2012'. International Journal of Human-Computer Studies, 74, 81-92.

lacovides, I. and Cox, A.L. (2015) 'Moving beyond fun: Evaluating serious experience in digital games'. In Proceedings of the 33rd Annual ACM Conference on Human Factors in Computing Systems. New York: Association for Computing Machinery, 2245-54.

Iversen, O.S., Halskov, K. and Leong, T.W. (2010) 'Rekindling values in participatory design'. In Proceedings of the 11th Biennial Participatory Design Conference. New York: Association for Computing Machinery, 91-100.

Jennett, C., Furniss, D., lacovides, I., Wiseman, S., Gould, S.J.J. and Cox, A.L. (2014) 'Exploring citizen psych-science and the motivations of Errordiary volunteers'. Human Computation, 1 (2), 201-20.

Jennett, C., Kloetzer, L., Himmelstein, J., Vaugoux, A., lacovides, I. and Cox, A.L. (2016) 'Learning in game jams: A case study of the GLASS Summer School'. Session presented the Playful Learning Conference, Manchester, 13-15 July 2016.

Kafai, Y.B. and Burke, Q. (2016) Connected Gaming: What making video games can teach us about learning and literacy. Cambridge, MA: MIT Press.

Kelty, C., Panofsky, A., Currie, M., Crooks, R., Erickson, S., Garcia, P., Wartenbe, M. and Wood, S. (2015) 'Seven dimensions of contemporary participation disentangled'. Journal of the Association for Information Science and Technology, 66 (3), 474-88.

Khaled, R. and Vasalou, A. (2014) 'Bridging serious games and participatory design'. International Journal of Child-Computer Interaction, 2 (2), 93-100.

Khatri, N., Brown, G.D. and Hicks, L.L. (2009) 'From a blame culture to a just culture in health care'. Health Care Management Review, 34 (4), 312-22.

Lam, B. (2013) 'Community-led design through digital games'. Design Management Institute Review, 24 (1), 20-7.

Li, Q. (2010) 'Digital game building: Learning in a participatory culture'. Educational Research, $52(4), 427-43$

Lukosch, H., van Ruijven, T. and Verbraeck, A. (2012) 'The participatory design of a simulation training game'. In Laroque, C., Himmelspach, J., Pasupathy, R., Rose, O. and Uhrmacher, A.M. (eds) Proceedings of the 2012 Winter Simulation Conference. Piscataway, NJ: Institute of Electrical and Electronics Engineers, 1-11.

Mahony, N. and Stephansen, H.C. (2016) 'The frontiers of participatory public engagement'. European Journal of Cultural Studies, 19 (6), 583-97. 
Marsh, T. and Costello, B. (2013) 'Lingering serious experience as trigger to raise awareness, encourage reflection and change behavior'. In Berkovsky, S. and Freyne, J. (eds) Persuasive Technology: 8th International Conference, PERSUASIVE 2013, Sydney, NSW, Australia, April 3-5, 2013 Proceedings. Heidelberg: Springer, 116-24.

Moser, C., Tscheligi, M., Magnusson, M. and Mueller, F. (2014) 'Game idea jam for sport and exertion games'. In Proceedings of the First ACM SIGCHI Annual Symposium on ComputerHuman Interaction in Play. New York: Association for Computing Machinery, 461-4.

Musil, J., Schweda, A., Winkler, D. and Biffl, S. (2010) 'Synthesized essence: What game jams teach about prototyping of new software products'. In Proceedings of the 32nd ACM/IEEE International Conference on Software Engineering (Vol. 2). New York: Association for Computing Machinery, 183-6.

NCCPE (National Co-ordinating Centre for Public Engagement) (2018) 'What is public engagement?'. Online. https://tinyurl.com/mx5og3g (accessed 4 November 2018).

Neresini, F. and Bucchi, M. (2011) 'Which indicators for the new public engagement activities? An exploratory study of European research institutions'. Public Understanding of Science, $20(1), 64-79$.

Ouariachi, T., Olvera-Lobo, M.D. and Gutiérrez-Pérez, J. (2017) 'Analyzing climate change communication through online games: Development and application of validated criteria'. Science Communication, 39 (1), 10-44.

Reason, J. (2000) 'Human error: Models and management'. British Medical Journal, 320 (7237), 768-70.

Reng, L., Schoenau-Fog, H. and Kofoed, L.B. (2013) 'The motivational power of game communities - engaged through game jamming'. In Workshop Proceedings of the 8th International Conference on the Foundations of Digital Games. Santa Cruz, CA: Society for the Advancement of the Science of Digital Games, 1-8. Online. https://tinyurl.com/y7ucyw9w (accessed 4 November 2018).

Resnick, M., Maloney, J., Monroy-Hernández, A., Rusk, N., Eastmond, E., Brennan, K., Millner, A., Rosenbaum, E., Silver, J., Silverman, B. and Kafai, Y. (2009) 'Scratch: Programming for all'. Communications of the ACM, 52 (11), 60-7.

Sayer, E.J., Featherstone, H.C., Gosling, W.D. and the BES Roadies (2014) 'Sex and bugs and rock 'n roll: Getting creative about public engagement'. Trends in Ecology and Evolution, 29 (2), 65-7.

Scanlon, E. (2014) 'Scholarship in the digital age: Open educational resources, publication and public engagement'. British Journal of Educational Technology, 45 (1), 12-23.

Shapiro, A., McDonald, B. and Johnston, A. (2014) 'Gathering the voices: Disseminating the message of the Holocaust for the digital generation by applying an interdisciplinary approach'. Social Sciences, 3 (3), 499-513.

Vasalou, A., Ingram, G. and Khaled, R. (2012) 'User-centered research in the early stages of a learning game'. In Proceedings of the Designing Interactive Systems Conference. New York: Association for Computing Machinery, 116-25.

Vines, J., Clarke, R., Wright, P., McCarthy, J. and Olivier, P. (2013) 'Configuring participation: On how we involve people in design'. In Proceedings of the SIGCHI Conference on Human Factors in Computing Systems. New York: Association for Computing Machinery, 429-38.

Watermeyer, R. (2012) 'From engagement to impact? Articulating the public value of academic research'. Tertiary Education and Management, 18 (2), 115-30.

Yin, R.K. (2009) Case Study Research: Design and methods. 4th ed. Thousand Oaks, CA: SAGE Publications. 